\title{
Full width at half maximum (FWHM) analysis of solitonic pulse applicable in optical network communication
}

\author{
IS Amiri ${ }^{1, ~ *, ~ H . ~ A h m a d ~}{ }^{1}$, Hamza M. R. Al-Khafaji² \\ ${ }^{1}$ Photonics Research Centre, University of Malaya (UM), 50603 Kuala Lumpur, Malaysia \\ ${ }^{2}$ Wireless Communication Centre, Faculty of Electrical Engineering, Universiti Teknologi Malaysia (UTM), 81310 UTM Skudai, Johor, \\ Malaysia
}

\author{
Email address: \\ amiri@um.edu.my (I. Amiri), harith@um.edu.my (H. Ahmad), hamza@utm.my (H. M. R. Al-Khafaji)
}

\section{To cite this article:}

IS Amiri, H. Ahmad, Hamza M. R. Al-Khafaji. Full Width at Half Maximum (FWHM) Analysis of Solitonic Pulse Applicable in Optical Network Communication. American Journal of Networks and Communications. Special Issue: Recent Progresses in Optical Code-Division Multiple-Access (OCDMA) Technology. Vol. 4, No. 2-1, 2015, pp. 1-5. doi: 10.11648/j.ajnc.s.2015040201.11

\begin{abstract}
In this paper, we propose a system of microring resonator (MRR). This system uses a laser diode input which can be incorporated with an optical add/drop filter system. When light from the laser diode feedbacks to the fiber ring resonator, the pulses in the form of soliton can be generated by using appropriate fiber ring resonator parameters and also the input power. The filtering process occurs during the propagation of the pulse within the ring resonators. The full width at half maximum (FWHM) or bandwidth characterization of the pulse can be performed using the proposed system. Results obtained have established particular possibilities from the application such as optical network communication. The obtained results show the effects of coupling coefficients and ring radius on the bandwidth of the soliton pulse, where the graph of the FWHM versus the variable parameters such as the radius and coupling coefficient are presented.
\end{abstract}

Keywords: Optical Network Communication, Soliton, FWHM, Pulse Bandwidth Characterization

\section{Introduction}

In optical communication, soliton controls within a semiconductor add/drop filter have numerous applications [1, 2]. Microring resonators (MRRs) are the types of Fabry-Pérot resonators, which can be readily integrated in array geometries for useful functions in areas such as optical communication [3, 4], signal processing in the nanoscale regime [5]. Its nonlinear phase response can also be readily incorporated into an interferometer system to produce a specific intensity output function [6, 7]. One interesting result emerges through the use of an add/drop system, which is a good candidate for nanoscale interferometer applications [8, 9]. One new feature of this specific type of ring resonator, which introduces a system of nanoscale-sensing transducers based on the add/drop ring resonator was presented by Amiri et al $[10,11]$. They have shown that the multisoliton can be generated and controlled within a modified add/drop ring resonator $[12,13]$.

Nonlinear behaviors associated with light traveling inside a fiber optic ring resonator can be caused by the effects such as the Kerr effects, four-wave mixing, as well as the external nonlinear pumping electrical power $[14,15]$. This sort of nonlinear behaviors usually are called chaos, bistability, in addition to bifurcation. Additional information regarding these kinds of behaviors in a micro ring resonator evidently are defined by Amiri et al $[16,17]$. Nonetheless, aside from the penalties of the nonlinear behaviors of light traveling within the fiber ring resonator, there are several benefits that can be employed by the communication methods in order to examine the obtained result. One called chaotic behavior which has been employed to make the benefit within digital or optical communications $[18,19]$. In this paper we consider the characterization of soliton pulses using MRRs. The bandwidth and free spectrum range of the soliton pulses can be manipulated using suitable parameters of the system.

\section{Theory of The Research}

The system of the ring resonator interferometer is shown in Figure 1. 


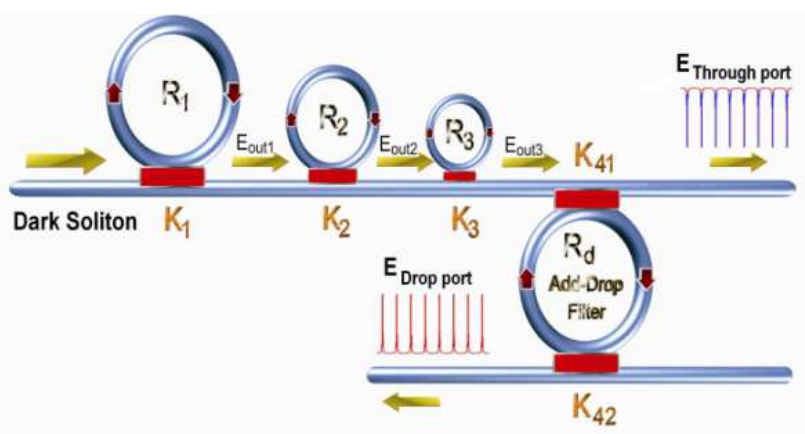

Figure 1. A schematic of the proposed MRR's system, where $R_{s}$ : ring radii, $\kappa_{s}$ : coupling coefficients, $R_{d}$ : an add/drop ring radius, $A_{\text {effs: }}$ effective areas

The input optical fields $\left(E_{\text {in }}\right)$ in the form of dark soliton can be expressed by [20]

$$
E_{\text {in }}(t)=A \tanh \left[\frac{T}{T_{0}}\right] \exp \left[\left(\frac{i z}{2 L_{D}}\right)-i \omega_{0} t\right]
$$

Here $\mathrm{A}$ and $\mathrm{z}$ are the optical field amplitude and propagation distance, respectively [21]. $T$ represents the soliton pulse propagation time in a frame moving at the group velocity, $\left(T=t-\beta_{1} \times z\right)$, where $\beta_{1}$ and $\beta_{2}$ are the coefficients of the linear and second order terms of the Taylor expansion of the propagation constant [22-24]. The dispersion length of the soliton pulse can be defined as $L_{D}=T_{0}^{2} /\left|\beta_{2}\right|$, where the frequency carrier of the soliton is $\omega_{0}$ [25]. The intensity of soliton peak is $\left(\left|\beta_{2} / \Gamma T_{0}^{2}\right|\right)$, where $T_{o}$ is representing the initial soliton pulse propagation time $[26$, 27]. A balance should be achieved between the dispersion length $\left(L_{D}\right)$ and the nonlinear length $\left(L_{N L}=1 / \Gamma \phi_{N L}\right)$, where $\Gamma=n_{2} \times k_{0}$, is the length scale over which disperse or nonlinear effects causes the beam becomes wider or narrower. Here, $L_{D}=L_{N L}$ [28]. The total index (n) of the system is given by $[29,30]$.

$$
n=n_{0}+n_{2} I=n_{0}+\left(\frac{n_{2}}{A_{e f f}}\right) P,
$$

where $n_{0}$ and $n_{2}$ are the linear and nonlinear refractive indices, respectively. $I$ and $P$ are the optical intensity and optical power, respectively $[31,32] . A_{\text {eff }}$ represents the effective mode core area of the device, where in the case of MRRs, the effective mode core areas range from 0.50 to 0.1 $\mu \mathrm{m} 2$. The normalized output of the light field is defined as $[33,34]$.

$$
\left|\frac{E_{\text {out }}(t)}{E_{\text {in }}(t)}\right|^{2}=(1-\gamma) \times\left[1-\frac{\left(1-(1-\gamma) x^{2}\right) \kappa}{(1-x \sqrt{1-\gamma} \sqrt{1-\kappa})^{2}+4 x \sqrt{1-\gamma} \sqrt{1-\kappa} \sin ^{2}\left(\frac{\phi}{2}\right)}\right]
$$

Here, $\boldsymbol{K}$ is the coupling coefficient, $x=\exp (-\alpha L / 2)$ represents a round-trip loss coefficient, $\phi=\phi_{0}+\phi_{N L}, \phi_{0}=k L n_{0}$ and $\phi_{N L}=k L n_{2}\left|E_{i n}\right|^{2}$ are the linear and nonlinear phase shifts and $k=2 \pi / \lambda$ is the wave propagation number and $\gamma$ is the fractional coupler intensity loss $[35,36]$. Here $L$ and $\alpha$ are the waveguide length and linear absorption coefficient, respectively $[37,38]$. The input power insert into the input port of the add/drop filter system. $E_{t h}$ and $E_{\text {drop }}$ represent the optical electric fields of the through and drop ports, respectively expressed by equations (4) and (5) [39, 40],

$$
\begin{aligned}
& \left|E_{\text {th }}\right|^{2} /\left|E_{\text {out }}\right|^{2}=\frac{\left(1-\kappa_{41}\right)-2 \sqrt{1-\kappa_{41}} \cdot \sqrt{1-\kappa_{42}} e^{-\frac{\alpha}{2} L_{d}} \cos \left(k_{n} L_{d}\right)+\left(1-\kappa_{42}\right) e^{-\alpha L_{d}}}{1+\left(1-\kappa_{41}\right)\left(1-\kappa_{42}\right) e^{-\alpha L_{d}}-2 \sqrt{1-\kappa_{41}} \cdot \sqrt{1-\kappa_{42}} e^{-\frac{\alpha}{2} L_{d}} \cos \left(k_{n} L_{d}\right)} \\
& \left|E_{\text {drop }}\right|^{2} /\left|E_{\text {out }}\right|^{2}=\frac{\kappa_{41} \kappa_{42} e^{-\frac{\alpha}{2} L_{d}}}{1+\left(1-\kappa_{41}\right)\left(1-\kappa_{42}\right) e^{-\alpha L_{d}}-2 \sqrt{1-\kappa_{41}} \cdot \sqrt{1-\kappa_{42}} e^{-\frac{\alpha}{2} L_{d}} \cos \left(k_{n} L_{d}\right)}
\end{aligned}
$$

where $\left|E_{t h}\right|^{2}$ and $\left|E_{\text {drop }}\right|^{2}$ are the output intensities of the through and drop ports respectively [41, 42].

\section{Results and Discussion}

Temporal profile of the input dark soliton pulse can be seen from Figure 2, where the input of the pulse is $350 \mathrm{~mW}$ with central wavelength of $1.3 \mu \mathrm{m}$. The ring radii of the rings are selected to $R_{1}=30 \mu \mathrm{m}, R_{2}=12 \mu \mathrm{m}$ and $R_{3}=5 \mu \mathrm{m}$, where $\kappa_{1}=0.7, \kappa_{2}=0.9$ and $\kappa_{3}=0.93$.

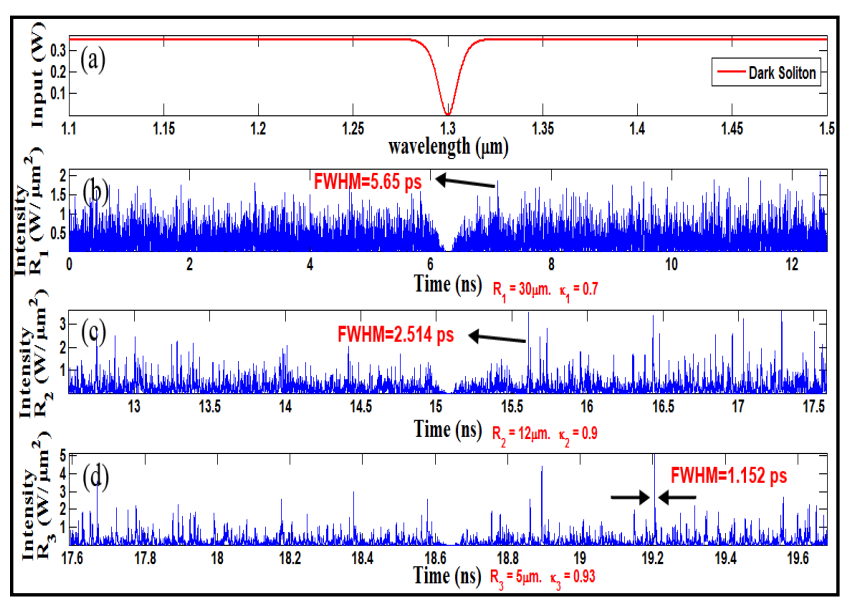

Figure 2. Results of temporal chaotic signals generation within a series of MRRs with dark soliton input

In Figure 2(a), the dark soliton is input and propagates within the ring system. In Figure 2(b), the input pulse is split to many noisy and chaotic signals in the form of temporal signals with $\mathrm{FWHM}=5.65 \mathrm{ps}$, where Figures 2(c) and 2(d) show the temporal signals seen within the range of 12.5-17.5 ns and 17.6-19.6 ns with FWHM of 2.514 and 1.152 ps respectively. The shorter bandwidth of the chaotic signals can be obtained by adding more ring resonators. The effects of the ring's radius and coupling coefficients of the ring resonators on the FWHM of the chaotic pulses are shown by Figure 3.

Therefore, the chaotic signals can be generated using the input dark soliton pulse. In order to use the chaotic signals for long distance communication, the use of input bright 
soliton is recommended, where the security of soliton signals can be performed when the input dark soliton is inserted into the system and split into chaotic signals.

Optical field of the Gaussian pulse can be inserted into the input port of the multi-stage MRR's system shown in Figure 4. Considering the proposed system, the radii of the rings have been selected as $R_{1}=15 \mu \mathrm{m}, R_{2}=9 \mu \mathrm{m}, R_{3}=7 \mu \mathrm{m}$, and $\kappa_{1}=0.96, \kappa_{2}=0.94, \kappa_{3}=0.92$, where the add/drop filter has a radius of $R_{d}=78 \mu \mathrm{m}$ and coupling coefficients of $\kappa_{4}=\kappa_{5}=0.1$. Some parameters of the system are fixed such as $n_{0}=3.34$ (InGaAsP/InP), $A_{e f f}=0.50,0.25$ and $0.10 \mu \mathrm{m}^{2}$ for the microrings, $\alpha=0.5 \mathrm{dBmm}^{-1}, \gamma=0.1$. The nonlinear refractive index of the MRRs is $n_{2}=2.2 \times 10^{-17} \mathrm{~m}^{2} / \mathrm{W}$.
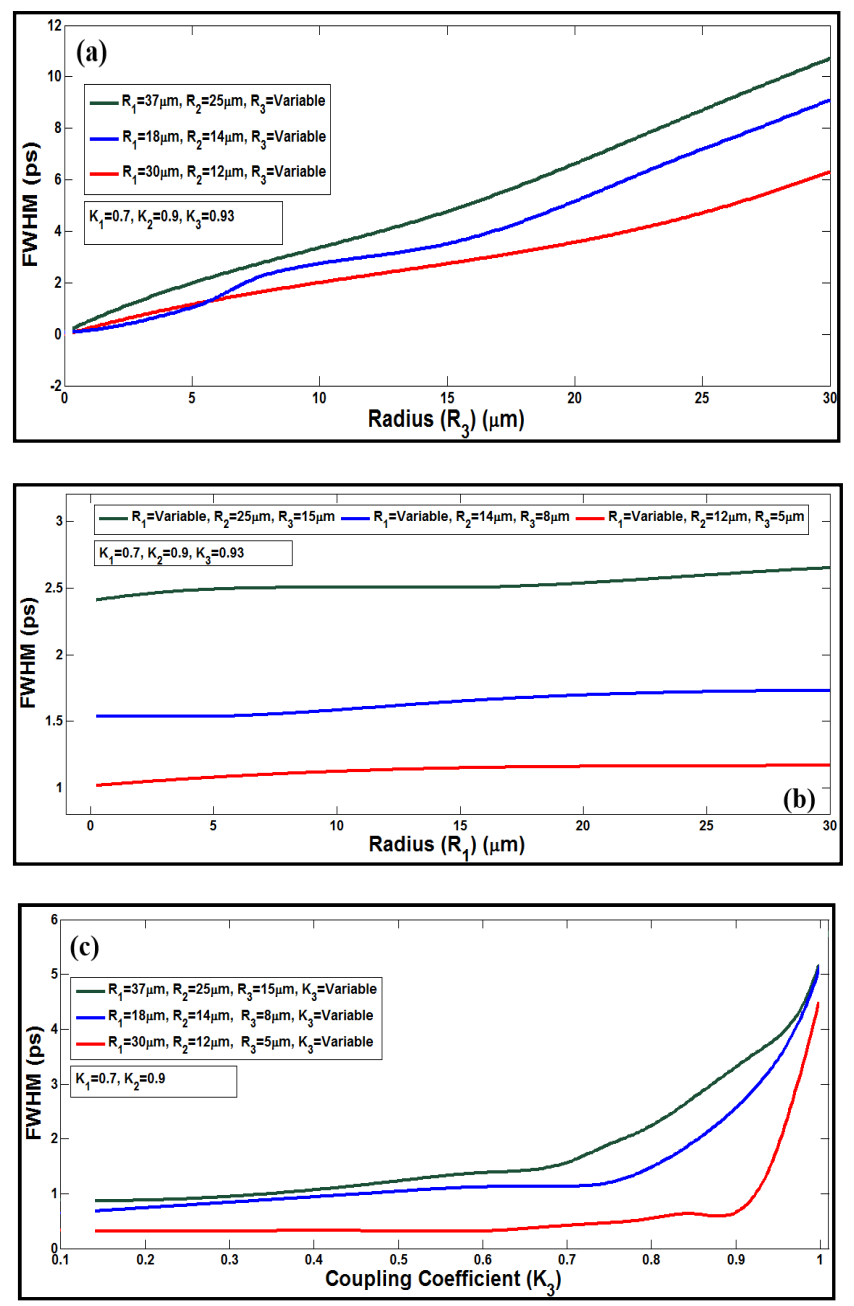

Figure 3. Results of FWHM, where (a): radius of the third ring varies, (b): radius of the first ring varies, (c): coupling coefficient of the third ring varies

The input Gaussian laser pulse with power of $2 \mathrm{~W}$ is introduced into the MRR's system shown in Figure 4(a). The output powers from three ring resonators are shown in Figures 4(b-d), where Figures 4(e-f) show the output power from the throughput port in terms of wavelength. The FWHM and FSR of the spatial soliton pulses are $50 \mathrm{pm}$ and $1440 \mathrm{pm}$ respectively. Figures $4(\mathrm{~g}-\mathrm{h})$ show the output power from the drop port of the system with the same FWHM and FSR.

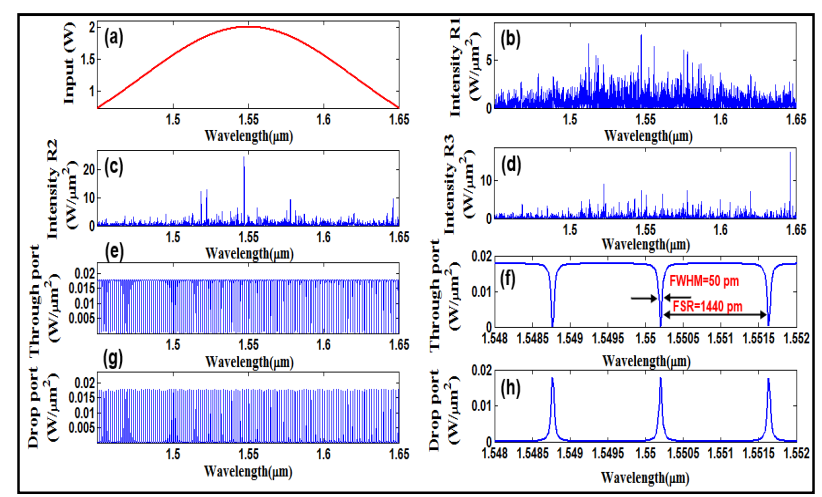

Figure 4. Results of spatial dark and bright soliton generation, where (a): input Gaussian beam, (b-d): large bandwidth signals, (e-f): dark soliton at the through port with FWHM and FSR of $50 \mathrm{pm}$ and $1440 \mathrm{pm}$ respectively, (g-h): bright soliton at the drop port with FWHM and FSR of $50 \mathrm{pm}$ and 1440 pm respectively

The advantage of this technique is its ability to operate on the trains of low-power picometer optical pulses. In order to improve the system, narrower soliton pulses are recommended, where the attenuation of such signals during transmission lessens when compared to the conventional peaks of micrometre laser pulses. Therefore, the bandwidth varies with respect to the variation of the coupling coefficients of the add/drop filter system shown in Figure 5.

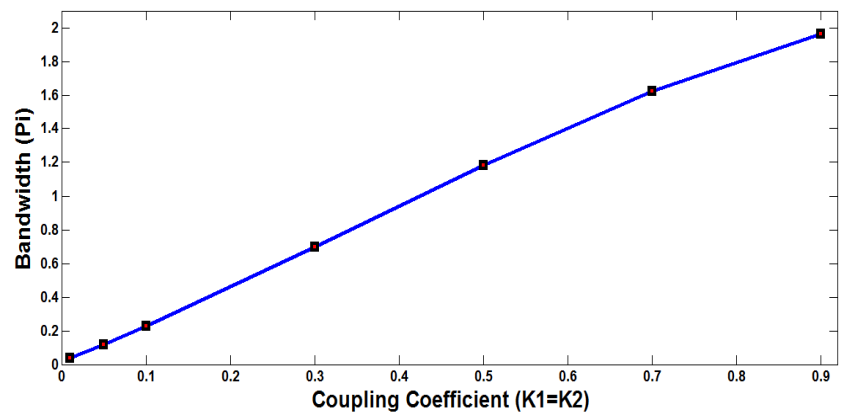

Figure 5. Result of the single bandwidth manipulation respect to variation of the coupling coefficients of the add/drop filter system

As it can be seen from the Figure 5, the increase of the coupling coefficients of the add/drop filter system leads to increase of the bandwidth of the soliton pulse. Thus in order to use ultra-short soliton pulses applied in optical communication, lower coupling coefficient is recommended where, the power control can be performed within the system [43-45].

Using this method, the output power of the system can be simulated successfully. This system act as a passive filter system which can be used to split the input power and generate chaotic signals using suitable parameters of the system [46-48]. Therefore input power of Gaussian beam can be sliced to smaller peaks as chaotic signals. The chaotic signals have many applications in optical communications.

\section{Conclusion}

Soliton signals can be generated using the input laser 
power propagating within a nonlinear ring resonator, where the required signals can be recovered and manipulated by using appropriate parameters of the system such as the ring radius and coupling coefficients. Results obtained have shown that the FWHM of the generated soliton pulses can be affected by vary the parameters. Here, the effects of coupling coefficients and radius on the bandwidth of the soliton pulse have been presented.

\section{References}

[1] Iraj Sadegh Amiri, Sayed Ehsan Alavi \& Sevia Mahdaliza Idrus, Soliton Coding for Secured Optical Communication Link. USA: Springer, 2014.

[2] IS Amiri \& A Afroozeh, Soliton Generation Based Optical Communication, in Ring Resonator Systems to Perform Optical Communication Enhancement Using Soliton, ed USA: Springer, 2014

[3] I. S. Amiri, S. E. Alavi, H. Ahmad, A.S.M. Supa'at \& N. Fisal, (2014) "Numerical Computation of Solitonic Pulse Generation for Terabit/Sec Data Transmission", Optical and Quantum Electronics,

[4] IS Amiri, MZ Zulkifli \& H Ahmad, (2014) "Soliton comb generation using add-drop ring resonators", International Research Journal of Telecommunications and Information Technology,

[5] Iraj Sadegh Amiri, Sayed Ehsan Alavi, S. M. Idrus, Abdolkarim Afroozeh \& Jalil Ali, Soliton Generation by Ring Resonator for Optical Communication Application. Hauppauge, NY 11788 USA: Nova Science Publishers, 2014.

[6] Amiri, H Ahmad \& MZ Zulkifli, (2014) "Integrated ring resonator system analysis to Optimize the soliton transmission", International Research Journal of Nanoscience and Nanotechnology, 1(1), 002-007.

[7] Iraj Sadegh Amiri \& Harith Ahmad, Optical Soliton Communication Using Ultra-Short Pulses. USA: Springer, 2014.

[8] I. S. Amiri, S. E. Alavi, Sevia M. Idrus, A. Nikoukar \& J. Ali, (2013) "IEEE 802.15.3c WPAN Standard Using Millimeter Optical Soliton Pulse Generated By a Panda Ring Resonator", IEEE Photonics Journal, 5(5), 7901912.

[9] A. Afroozeh, A.Zeinalinezhad, I. S. Amiri \& S. E. Pourmand, (2014) "Stop Light Generation using Nano Ring Resonators for ROM", Journal of Computational and Theoretical Nanoscience (CTN), 12(3),

[10] S. E. Alavi, I. S. Amiri, S. M. Idrus, ASM Supa'at, J. Ali \& P. P. Yupapin, (2014) "All Optical OFDM Generation for IEEE802.11a Based on Soliton Carriers Using MicroRing Resonators ", IEEE Photonics Journal, 6(1),

[11] IS Amiri, SE Alavi, N Fisal, ASM Supa'at \& H Ahmad, (2014) "All-Optical Generation of Two IEEE802.11n Signals for $2 \times 2$ MIMO-RoF via MRR System", IEEE Photonics Journal, 6(6),

[12] I. S. Amiri \& J. Ali, (2014) "Generating Highly Dark-Bright Solitons by Gaussian Beam Propagation in a PANDA Ring Resonator", Journal of Computational and Theoretical Nanoscience (CTN), 11(4), 1092-1099.
[13] I. S. Amiri, S. E. Alavi \& J. Ali, (2013) "High Capacity Soliton Transmission for Indoor and Outdoor Communications Using Integrated Ring Resonators", International Journal of Communication Systems,

[14] IS Amiri, SE Alavi, H Ahmad, ASM Supa'at \& N Fisal, (2014) "Generation and Transmission of $3 \times 3$ W-Band MIMOOFDM-RoF Signals Using Micro-Ring Resonators", Applied Optics, 53(34),

[15] I. S. Amiri, M. Ranjbar, A. Nikoukar, A. Shahidinejad, J. Ali \& P. Yupapin, (2012), "Multi optical Soliton generated by PANDA ring resonator for secure network communication", in Computer and Communication Engineering (ICCCE) Conference, Malaysia, 760-764.

[16] I. S. Amiri, S. E. Alavi \& H. Ahmad, (2015) "Analytical Treatment of the Ring Resonator Passive Systems and Bandwidth Characterization Using Directional Coupling Coefficients ", Journal of Computational and Theoretical Nanoscience (CTN), 12(3),

[17] Iraj Sadegh Amiri \& Abdolkarim Afroozeh, Ring Resonator Systems to Perform the Optical Communication Enhancement Using Soliton. USA: Springer, 2014.

[18] IS Amiri \& A Afroozeh, Integrated Ring Resonator Systems, in Ring Resonator Systems to Perform Optical Communication Enhancement Using Soliton, ed USA: Springer, 2014.

[19] I. S. Amiri, S. Soltanmohammadi, A. Shahidinejad \& j. Ali, (2013) "Optical quantum transmitter with finesse of 30 at 800 $\mathrm{nm}$ central wavelength using microring resonators", Optical and Quantum Electronics, 45(10), 1095-1105.

[20] I. S. Amiri, A. Afroozeh, I. N. Nawi, M. A. Jalil, A. Mohamad, J. Ali \& P. P. Yupapin, (2011) "Dark Soliton Array for communication security", Procedia Engineering, 8 417-422.

[21] I. S. Amiri, S. E. Alavi, S. M. Idrus, A. S. M. Supa'at, J. Ali \& P. P. Yupapin, (2014) "W-Band OFDM Transmission for Radio-over-Fiber link Using Solitonic Millimeter Wave Generated by MRR", IEEE Journal of Quantum Electronics, $50(8), 622-628$.

[22] I. S. Amiri \& J. Ali, (2013) "Data Signal Processing Via a Manchester Coding-Decoding Method Using Chaotic Signals Generated by a PANDA Ring Resonator", Chinese Optics Letters, 11(4), 041901(4).

[23] S. E. Alavi, I. S. Amiri, M. R. K. Soltanian, A.S.M. Supa'at, N. Fisal \& H. Ahmad, (2015) "Generation of Femtosecond Soliton Tweezers Using a Half-Panda System for Modeling the Trapping of a Human Red Blood Cell", Journal of Computational and Theoretical Nanoscience (CTN), 12(1),

[24] I. S. Amiri, S. E. Alavi \& S. M. Idrus, Theoretical Background of Microring Resonator Systems and Soliton Communication, in Soliton Coding for Secured Optical Communication Link, ed USA: Springer, 2015, pp. 17-39.

[25] A. Afroozeh, I.S. Amiri, K. Chaudhary, J. Ali \& P. P. Yupapin, Analysis of Optical Ring Resonator, in Advances in Laser and Optics Research, ed New York: Nova Science, 2014.

[26] I. S. Amiri, J. Ali \& P. P. Yupapin, (2012) "Enhancement of FSR and Finesse Using Add/Drop Filter and PANDA Ring Resonator Systems", International Journal of Modern Physics B, 26(04), 1250034. 
[27] I. S. Amiri, B. Barati, P. Sanati, A. Hosseinnia, HR Mansouri Khosravi, S. Pourmehdi, A. Emami \& J. Ali, (2014) "Optical Stretcher of Biological Cells Using Sub-Nanometer Optical Tweezers Generated by an Add/Drop Microring Resonator System", Nanoscience and Nanotechnology Letters, 6(2), 111117.

[28] I. S. Amiri, S. E. Alavi \& S. M. Idrus, Introduction of Fiber Waveguide and Soliton Signals Used to Enhance the Communication Security, in Soliton Coding for Secured Optical Communication Link, ed USA: Springer, 2015, pp. 1-16.

[29] I. S. Amiri \& J. Ali, (2014) "Femtosecond Optical Quantum Memory generation Using Optical Bright Soliton", Journal of Computational and Theoretical Nanoscience (CTN), 11(6), $1480-1485$.

[30] I. S. Amiri \& J. Ali, (2013) "Nano Particle Trapping By Ultrashort tweezer and wells Using MRR Interferometer System for Spectroscopy Application", Nanoscience and Nanotechnology Letters, 5(8), 850-856.

[31] P. Sanati, A. Afroozeh, I. S. Amiri, J.Ali \& Lee Suan Chua, (2014) "Femtosecond Pulse Generation using Microring Resonators for Eye Nano Surgery", Nanoscience and Nanotechnology Letters, 6(3), 221-226

[32] I. S. Amiri \& J. Ali, (2014) "Optical Quantum Generation and Transmission of 57-61 GHz Frequency Band Using an Optical Fiber Optics ", Journal of Computational and Theoretical Nanoscience (CTN), 11(10), 2130-2135.

[33] S. E. Alavi, I. S. Amiri, S. M. Idrus \& A. S. M. Supa'at, (2014) "Generation and Wired/Wireless Transmission of IEEE $802.16 \mathrm{~m}$ Signal Using Solitons Generated By Microring Resonator", Optical and Quantum Electronics,

[34] S. E. Alavi, I. S. Amiri, H. Ahmad, N. Fisal \& ASM. Supa'at, (2015) "Optical Amplification of Tweezers and Bright Soliton Using an Interferometer Ring Resonator System", Journal of Computational and Theoretical Nanoscience (CTN), 12(4),

[35] I. S. Amiri, A. Nikoukar, A. Shahidinejad, J. Ali \& P. Yupapin, (2012), "Generation of discrete frequency and wavelength for secured computer networks system using integrated ring resonators", in Computer and Communication Engineering (ICCCE) Conference, Malaysia, 775-778.

[36] I. S. Amiri \& J. Ali, (2014) "Picosecond Soliton pulse Generation Using a PANDA System for Solar Cells Fabrication", Journal of Computational and Theoretical Nanoscience (CTN), 11(3), 693-701.

[37] I. S. Amiri, A. Nikoukar \& J. Ali, (2013) "GHz Frequency Band Soliton Generation Using Integrated Ring Resonator for WiMAX Optical Communication", Optical and Quantum Electronics, 46(9), 1165-1177.
[38] I. S. Amiri, M. Ebrahimi, A. H. Yazdavar, S. Gorbani, S. E. Alavi, Sevia M. Idrus \& J. Ali, (2014) "Transmission of data with orthogonal frequency division multiplexing technique for communication networks using $\mathrm{GHz}$ frequency band soliton carrier", IET Communications, 8(8), 1364 - 1373.

[39] I. S. Amiri, K. Raman, A. Afroozeh, M. A. Jalil, I. N. Nawi, J. Ali \& P. P. Yupapin, (2011) "Generation of DSA for security application", Procedia Engineering, 8 360-365.

[40] I. S. Amiri, P. Naraei \& J. Ali, (2014) "Review and Theory of Optical Soliton Generation Used to Improve the Security and High Capacity of MRR and NRR Passive Systems", Journal of Computational and Theoretical Nanoscience (CTN), 11(9), $1875-1886$.

[41] I. Sadegh Amiri, M. Nikmaram, A. Shahidinejad \& J. Ali, (2013) "Generation of potential wells used for quantum codes transmission via a TDMA network communication system", Security and Communication Networks, 6(11), 1301-1309.

[42] I. S. Amiri, A. Afroozeh \& M. Bahadoran, (2011) "Simulation and Analysis of Multisoliton Generation Using a PANDA Ring Resonator System", Chinese Physics Letters, 28(10), 104205 .

[43] Y. S. Neo, S. M. Idrus, M. F. Rahmat, S. E. Alavi \& I. S. Amiri', (2014) "Adaptive Control for Laser Transmitter Feedforward Linearization System", IEEE Photonics Journal 6(4),

[44] I. S. Amiri, S. E. Alavi, M. Bahadoran, A. Afroozeh \& H. Ahmad, (2015) "Nanometer Bandwidth Soliton Generation and Experimental Transmission within Nonlinear Fiber Optics Using an Add-Drop Filter System", Journal of Computational and Theoretical Nanoscience (CTN), 12(2),

[45] S. E. Alavi, I. S. Amiri, M. Khalily, A. S. M. Supa' at, N. Fisal, H. Ahmad \& S. M. Idrus, (2014) "W-Band OFDM for Radioover-Fibre Direct-Detection Link Enabled By Frequency Nonupling Optical Up-Conversion", IEEE Photonics Journal $6(6)$,

[46] I. S. Amiri, R. Ahsan, A. Shahidinejad, J. Ali \& P. P. Yupapin, (2012) "Characterisation of bifurcation and chaos in silicon microring resonator", IET Communications, 6(16), 2671-2675.

[47] S. E. Alavi, I.S.Amiri, A. S. M. Supa'at \& S. M. Idrus, (2015) "Indoor Data Transmission Over Ubiquitous Infrastructure of Powerline Cables and LED Lighting", Journal of Computational and Theoretical Nanoscience (CTN), 12(4).

[48] I. S. Amiri \& A. Afroozeh, Spatial and Temporal Soliton Pulse Generation By Transmission of Chaotic Signals Using Fiber Optic Link in Advances in Laser and Optics Research. vol. 11, ed New York: Nova Science Publisher, 2014. 